\title{
¿TIENEN FUTURO LOS MODELOS DSGE?
}

Olivier Blanchard*

Tos modelos de equilibrio general dinámico estocástico (DSGE) hoy Algunos los consideran como un indicio de que la macroeconomía se ha convertido en una ciencia madura, organizada en torno a un núcleo microfundamentado común. $\mathrm{Y}$ otros, como un peligroso callejón sin salida.

Creo que la primera afirmación es exagerada y que la segunda es errónea. Considero que los modelos DSGE actuales tienen graves defectos, pero que son sumamente mejorables y centrales para el futuro de la macroeconomía. Pero para mejorarlos tienen que volverse menos insulares, recurriendo a un conjunto mucho más amplio de investigación económica. También deben volverse menos imperialistas y aceptar compartir el escenario con otros enfoques de la modelación.

Para quienes no son macroeconomistas, o para los macroeconomistas que vivieron en una isla desierta durante los últimos veinte años, he aquí un breve repaso. DSGE significa "equilibrio general dinámico estocástico”. Los modelos DSGE son en efecto dinámicos y estocásticos,

* Fred Bergsten Senior Fellow en el Instituto Peterson de Economía Internacional. Profesor emérito de economía en el Miт, [blanchar@mit.edu]. Los puntos de vista que aquí se expresan son del autor y no necesariamente reflejan los puntos de vista de la Junta Directiva, el personal o la administración del Instituto. Agradezco a Tam Bayoumi, Bill Cline, Chris Erceg, Patrick Honohan, Narayana Kocherlakota, Jesper Lindé, Thomas Philippon, David Stockton, Ángel Ubide, Nicolas Véron y David Vines por sus comentarios y sugerencias a un borrador anterior. Tomado de Policy Brief, Peterson Institute for International Economics, agosto de 2016. Traducción de Alberto Supelano; se publica con las autorizaciones correspondientes. Fecha de recepción: 26-08-2016, fecha de aceptación: 20-10-2016. Sugerencia de citación: Blanchard, O. “'Tienen futuro los modelos DSGE?”, Revista de Economia Institucional 18, 35, 2016, pp. 39-46.

DOI: http://dx.doi.org/10.18601/01245996.v18n35.03. 
y caracterizan el equilibrio general de la economía. Para elaborarlos se toman tres decisiones estratégicas de modelación. Primera, cuando está presente, el comportamiento de consumidores, firmas e intermediarios financieros se deduce formalmente de microfundamentos. Segunda, el entorno económico subyacente es el de una economía competitiva, al que se añaden algunas distorsiones esenciales: rigideces nominales, poder de monopolio, problemas de información. Tercera, el modelo se estima como un sistema, y no ecuación por ecuación como en las generaciones anteriores de modelos macroeconómicos. El modelo DSGE más antiguo, que representaba una economía sin distorsiones, fue el modelo del ciclo real de los negocios desarrollado por Edward C. Prescott, centrado en los efectos de los choques de productividad. En encarnaciones posteriores cumplen un mayor papel conjuntos más amplios de distorsiones y de choques, y los modelos DSGE se perciben mejor como versiones a gran escala del modelo nuevo keynesiano, que subraya las rigideces nominales y el papel de la demanda agregada ${ }^{1}$.

Hay muchas razones para sentir disgusto por los modelos DSGE actuales:

Primera. Se basan en supuestos poco atractivos. No solo supuestos simplificadores, como debe hacerlo todo modelo, sino profundamente contrarios a lo que sabemos de los consumidores y las firmas.

Volvamos al modelo de referencia nuevo keynesiano estándar del que los DSGE derivan su estructura ósea. Ese modelo consta de tres ecuaciones: una que describe la demanda agregada, una que describe el ajuste de precios y una que describe la regla de política monetaria. Al menos las dos primeras son descripciones muy defectuosas de la realidad: la demanda agregada se infiere de la demanda de consumo de consumidores previsivos que tienen una vida infinita. Sus implicaciones con respecto al grado de previsión y al papel de las tasas de interés en la modificación de la trayectoria del consumo están en fuerte discordancia con la evidencia empírica. El ajuste de precios es caracterizado por una ecuación de inflación futura que no capta la inercia fundamental de la inflación ${ }^{2}$.

\footnotetext{
${ }^{1}$ Aunque no existe "el modelo DsGE estándar", la referencia estándar sigue siendo el modelo desarrollado por Frank Smets y Rafael Wouters (2007). Para una valoración reciente con muchas referencias, ver Lindé, Smets y Wouters (2016).

${ }^{2}$ Más específicamente, la ecuación que caracteriza el comportamiento de los consumidores es la condición de primer orden del problema de optimización correspondiente y se conoce como "ecuación de Euler". La ecuación que caracteriza el comportamiento de los precios se deriva de una formalización propuesta por Guillermo Calvo y se conoció "fijación de precios de Calvo".
} 
Los modelos DSGE actuales extienden el modelo nuevo keynesiano de muchas maneras: teniendo en cuenta la inversión y la acumulación de capital, la intermediación financiera, la interacción con otros países, etc. Las ecuaciones de demanda agregada y de ajuste de precios siguen siendo centrales, aunque modificadas para ajustarlas mejor a los datos. En el primer caso, considerando por ejemplo que una proporción de consumidores es de consumidores que "viven al día" y simplemente consumen su ingreso. En el segundo caso, introduciendo la indexación de precios hacia atrás que, casi como un supuesto, genera inercia de inflación. Pero ambos son remiendos y no caracterizaciones convincentes del comportamiento de los consumidores, de los precios y de quienes fijan los salarios.

Segunda. Su método usual de estimación, una mezcla de calibración y estimación bayesiana, no es convincente.

Los modelos se estiman como un sistema, y no ecuación por ecuación como en los modelos macroeconométricos anteriores. Sin embargo, se debe estimar un número de parámetros muy alto, y no es factible la estimación clásica de todo el conjunto. Por ello varios parámetros se fijan a priori, mediante “calibración”. Este enfoque sería razonable si esos parámetros estuviesen bien establecidos empírica o teóricamente. Por ejemplo, bajo el supuesto de que la función de producción es Cobb-Douglas puede ser razonable usar la participación del trabajo como exponente del trabajo en la función de producción. Pero la lista de parámetros elegidos mediante calibración suele ser mucho mayor, y la evidencia es a menudo muy confusa. Por ejemplo, ante grandes diferencias en el comportamiento de la inflación entre países, es muy sospechoso usar los mismos "parámetros estándar de Calvo" (los cuales determinan el efecto del desempleo en la inflación) en países distintos. En muchos casos, la decisión de confiar en un "conjunto estándar de parámetros" es solo una manera de trasladar a investigadores anteriores la carga de elegir los parámetros.

Los parámetros restantes se calculan mediante la estimación bayesiana del modelo completo. Los problemas son de dos tipos. Uno es común a toda estimación del sistema. La mala especificación de una parte del modelo afecta el cálculo de los parámetros de otras partes del modelo. Por ejemplo, la mala especificación de la demanda agregada puede llevar a estimaciones erróneas del ajuste de precios y salarios, etc. Y de un modo que es opaco para el lector. El otro problema proviene de la complejidad de pasar de los parámetros a los datos. La estimación clásica es de hecho imposible porque la función de probabilidad es muy plana en muchas dimensiones. Si tuviésemos 
precedentes justificadamente ajustados para los coeficientes la estimación bayesiana parecería ser la manera de proceder. Pero en muchos casos la justificación del precedente ajustado es débil en el mejor de los casos, y lo que se estima refleja el precedente del investigador más que la función de probabilidad ${ }^{3}$.

Tercera. Aunque los modelos se pueden usar formalmente para propósitos normativos, las implicaciones normativas no son convincentes.

Una gran fortaleza potencial de los modelos DSGE es que, en la medida en que se derivan de microfundamentos, se pueden usar no solamente para propósitos descriptivos sino también para propósitos normativos. De hecho, en muchas discusiones de política es engañoso centrarse únicamente en el Рів о en el crecimiento del рів: los efectos de la distribución, o las distorsiones que afectan la composición en vez del tamaño del producto, o los efectos de las políticas actuales sobre el producto futuro en vez del producto corriente, pueden ser tan importantes para el bienestar como los efectos del PIB corriente. Como atestigua la importancia de las discusiones sobre la creciente desigualdad en Estados Unidos o sobre la composición del producto entre inversión y consumo en China.

En la práctica, el problema es que la inferencia de efectos de bienestar depende de la manera de introducir las distorsiones en el modelo. Y, a menudo, por razones de carácter práctico, esas distorsiones se introducen de maneras que son analíticamente convenientes pero cuyas implicaciones de bienestar no son convincentes. Para dar un ejemplo concreto, en estos modelos los efectos desfavorables de la inflación sobre el bienestar dependen principalmente de sus efectos sobre la distribución de los precios relativos pues no todas las firmas ajustan los precios nominales al mismo tiempo. La investigación sobre los costos y beneficios de la inflación sugiere, sin embargo, una gama mucho más amplia de efectos de la inflación sobre la actividad y a su vez sobre el bienestar.

En un artículo reciente (Blanchard, Erceg y Lindé, 2016), después de examinar las implicaciones de bienestar de diversas políticas mediante una función de bienestar ad hoc que refleja las desviaciones del producto con respecto a su potencial y de la inflación con respecto a la meta, y mediante la función de bienestar derivada del modelo,

\footnotetext{
${ }^{3}$ En algunos casos, las estimaciones de máxima verosimilitud de los parámetros están bien identificadas, pero son muy poco plausibles sobre bases teóricas. En este caso, los precedentes bayesianos ajustados llevan a estimaciones más plausibles. Sin embargo, es claro que en este caso el problema proviene de una especificación incorrecta del modelo y que los precedentes bayesianos ajustados son de nuevo un remiendo y no una solución.
} 
saqué dos conclusiones. Primera, el ejercicio de derivar la función de bienestar internamente consistente fue útil para mostrar efectos de bienestar potenciales en los que no había pensado pero que había concluido ex post que podían ser relevantes. Segunda, que tenía más confianza en las conclusiones de la función de bienestar ad hoc.

Cuarta. Los modelos DSGE son malos dispositivos de comunicación.

Un artículo típico sobre modelos DSGE añade una distorsión particular al núcleo existente. Empieza con una deducción pesadamente algebraica del modelo, luego pasa a la estimación y termina con varias simulaciones dinámicas que muestran los efectos de la distorsión sobre las propiedades de equilibrio general del modelo.

Estas parecen ser las características de una ciencia madura: basarse en un cuerpo de ciencia bien entendido y aceptado, y explorar modificaciones y extensiones. $\mathrm{Y}$, de hecho, tener un núcleo común enriquece la discusión entre quienes realmente producen estos modelos y han adquirido, a través de muchas simulaciones, cierta idea de sus entresijos (dejando de lado que el núcleo común sea correcto, el asunto planteado en la primera crítica anterior). Pero, para el lector casual, es sumamente difícil entender lo que hace una distorsión particular sobre sí misma y cómo interactúa después con otras distorsiones en el modelo.

Todas estas objeciones son graves. ¿Se suman al argumento en favor de descartar los DSGE y explorar otros enfoques? No lo creo. Creo que los DSGE toman las decisiones estratégicas básicas correctas y que se pueden encarar los defectos actuales. Permítanme desarrollar estos dos temas.

La búsqueda de un núcleo analítico macroeconómico ampliamente aceptado, en el cual situar las discusiones y extensiones, puede ser una quimera, pero es un sueño que vale la pena continuar. Si es así, las tres principales decisiones de modelación de los DSGE son las correctas. Partir de microfundamentos explícitos es claramente esencial; ¿por dónde más empezar? Las ecuaciones ad hoc no servirán para ese propósito. Pensar en términos de un conjunto de distorsiones respecto de una economía competitiva implica mucho trabajo duro para pasar del modelo competitivo a una descripción razonablemente plausible de la economía. Pero, de nuevo, es difícil ver por qué otra parte empezar. Pasando a la estimación, también parece esencial estimar y calibrar el modelo como un sistema, y no ecuación por ecuación. La experiencia de los modelos anteriores ecuación por ecuación demuestra que sus propiedades dinámicas pueden ser contrarias a la dinámica real del sistema. 
Dicho esto, creo que la modelación DSGE tiene que evolucionar de dos maneras.

Primera. Tiene que volverse menos insular. Tomemos el ejemplo del consumo que discutimos antes. En vez de buscar reparaciones, los modelos DSGE se deberían basar en el inmenso trabajo sobre el comportamiento de los consumidores de las diversas ramas de la economía, desde la economía del comportamiento, a los trabajos empíricos con grandes datos, a la estimación del equilibrio parcial macro. Ese trabajo está en curso y debe continuar por sí mismo, sin preocuparse por la integración DSGE. (Nota para los editores de revistas: no se debería exigir que toda discusión de un nuevo mecanismo venga con un cierre completo de equilibrio general.) Pero este conjunto de trabajos se debe reunir para darnos un mejor modelo del comportamiento de los consumidores, y quizá una idea de sus implicaciones de equilibrio general con un cierre simplista de equilibrio general, y después y solo después integrarlo en los modelos DSGE. Esto llevaría a especificaciones más plausibles y a precedentes bayesianos más confiables, y considero que esto es lo que más hace falta. Aquí me he centrado en el consumo, pero lo mismo es válido para la fijación de precios y salarios, la inversión, la intermediación financiera, el tratamiento de las expectativas, etc. En suma, los DSGE deberían ser la arquitectura en la cual eventualmente se integren y discutan los resultados relevantes de los diversos campos de la economía. Hoy no lo son.

Segunda. Tiene que volverse menos imperialista. O, quizá más justamente, la profesión ( $y$, de nuevo, esta es una nota para los editores de las principales revistas) debe entender que se necesitan diferentes tipos de modelos para tareas diferentes.

Los modelos pueden tener diferentes grados de pureza teórica. En un extremo, la máxima pureza teórica es de hecho el nicho de los DSGE. Para estos modelos, ajustar estrechamente los datos es menos importante que la claridad de la estructura. Luego vienen los modelos utilizados para propósitos de política, por ejemplo, los modelos de los bancos centrales o las organizaciones internacionales. Estos deben ajustar los datos más estrechamente, y es probable que esto requiera en particular estructuras rezagadas más flexibles y menos microfundamentadas (un ejemplo es el modelo FRB/Us utilizado por la Reserva Federal, que parte de microfundamentos pero permite que los datos determinen la estructura dinámica de las diversas relaciones). Por último vienen los modelos utilizados para hacer predicciones. Puede ser que, para ese propósito, los modelos en forma reducida sigan venciendo a los modelos estructurales durante 
algún tiempo; quizá la pureza teórica sea por ahora un obstáculo más que una fortaleza.

Los modelos también pueden diferir en el grado de simplicidad. No todos deben ser explícitamente microfundamentados. Aunque parezca un alegato pro domo, creo firmemente que los modelos macro ad hoc, desde las diversas versiones del modelo Is-LM hasta el modelo Mundell-Fleming, tienen un papel importante que jugar en relación con los modelos DSGE. Pueden ser útiles río arriba, antes de la modelación DSGE, como primer corte para pensar en los efectos de una distorsión particular o una política particular. Pueden ser útiles río abajo, después de la modelación DSGE, para presentar las principales ideas del modelo de manera más clara y pedagógica. Aquí, de nuevo, hay espacio para una variedad de modelos, dependiendo del grado en que sean $a d$ hoc: se puede pensar, por ejemplo, en el modelo nuevo keynesiano como un híbrido, como una versión microfundamentada pero muy simplificada de DSGE más grandes. Alguien dijo que esos modelos ad hoc son más arte que ciencia, y pienso que es cierto. En las manos correctas, son arte hermoso, pero no todos los economistas pueden o deben ser artistas. Hay espacio para la ciencia y el arte. Como ponente a menudo he encontrado, por ejemplo, que podía resumir los resultados de un artículo DSGE en una gráfica simple. Había aprendido algo del modelo formal, pero podía (y como ponente se me permitía) presentar la idea básica en forma más simple que al autor del artículo. El modelo DSGE y los modelos ad hoc eran complementos, no sustitutos.

Volviendo al asunto inicial: sospecho que incluso los modeladores DSGE estarán de acuerdo en que los modelos DSGE actuales son defectuosos. Pero los modelos DSGE pueden satisfacer una necesidad importante en macroeconomía: of recer una estructura nuclear alrededor de la cual construir y organizar las discusiones. Para ello, sin embargo, deben basarse más en el resto de la macroeconomía y aceptar compartir el escenario con otros tipos de modelos de equilibrio general.

\section{REFERENCIAS BIBLIOGRÁFICAS}

1. Blanchard, O.; Ch. Erceg y J. Lindé. "Jump-starting the Euro Area recovery: Would a rise in core fiscal spending help the periphery?", NBer Macroeconomics Annual conference, Cambridge, Mass., NBER, 2016.

2. Lindé, J.; F. Smets y R. Wouters. "Challenges for Central Banks' macro models", Riksbank Research Paper Series 147, 2016, [papers. ssrn.com/sol3/papers.cfm? abstract_id=2780455]. 
3. Smets, F. y R. Wouters. "Shocks and frictions in us business cycles: A Bayesian Dsge approach", American Economic Review 97, 3, 2007, pp. 586-606, [www.aeaweb.org/articles?id=10.1257/aer.97.3.586]. 